Updated information was obtained on current number of biological children, marital status, and average weekly running distance in the prior 12 months, and the Motivations of Marathoners Scales was completed.

Results.-Among the 1349 runners receiving the question, 349 (25.9\%) answered "yes." Those answering "yes" compared with those answering "no" were older (median age, 47.3 vs 43.3 years, $P<.0001$ ), more likely to be married $(76.2 \%$ vs $69.6 \%, P=.019$ ), had more children (median number 2 vs $1, P=.0095$ ), and ran less (median 48 vs $56 \mathrm{~km} / \mathrm{wk}$ in past year, $P<.0001$ ), but did not differ in sex (70.5\% vs $67.6 \%$ men, $P=.35$ ). The Motivations of Marathoners Scales showed significant group differences with those answering "yes" to the question having a higher health orientation (median 5.7 vs 5.3, $P<.0001$ ), and lower personal goal achievement (median 5.0 vs 5.2, $P=.0061$ ), psychological coping (median 4.4 vs $4.8, P<.0001$ ) and life meaning (median 4.6 vs $4.9, P=.0002$ ) scores.

Conclusion.-Ultramarathon runners find benefit from participating in ultramarathon running to the extent that most indicate they would not stop doing it even if it was bad for their health. Not surprisingly, those indicating they would stop were older, more likely to be married, had more children, were running less, were more health oriented, were less achievement oriented, and had less psychological motivations for running.

Supported by the Western States Endurance Run Foundation.

\section{The Development and Initial Assessment of a Novel Heart Rate Training Formula}

Tracy B. Høeg, MD $\mathrm{PhD}^{1}$; Phil Maffetone, $\mathrm{DC}^{2}$

${ }^{1}$ Nastved, Denmark, ${ }^{2}$ Oracle, AZ, USA

Objective.-To present the initial data behind a running gait-determined training heart rate and the resulting 180-Formula as an exercise intensity guide.

Methods.-In group 1, 223 male and female noninjured, experienced adult runners underwent extensive clinical evaluation and running gait analysis to determine the highest heart rate associated with an optimal running gait. Athletes were assigned training heart rates just below this and told to maintain their previous weekly mileage at or below their assigned heart rate. Pre- and post-study 5 -km races were performed on certified courses. In group 2, 38 participants were assigned a training heart rate using the methods above, while 39 controls maintained their normal training schedule.

Results.-In group 1, 223 of $225(99.1 \%)$ runners completed the program; 170 of 223 (76.2\%; 95\% confidence interval: 70.6-81.9\%) runners improved their $5-\mathrm{km}$ race times. In group 2,42 of 42 completed the program. The monitor group had an injury rate of 2 of 21 runners $(9.5 \%)$ and the control group had an injury rate of 13 of $21(61.9 \%)(P=.001)$. The assigned heart rates of these 2 groups of athletes were used to create a formula, called the 180-Formula, for use as a training intensity guide.

Conclusions. - The described training method and corresponding 180Formula appear to be a safe and effective gauge of exercise intensity. The target heart rate training method studied here deserves further exploration in endurance athletics and rehabilitation.

\section{Medical Care and Runner Characteristics at a 161-km High Altitude Ultraendurance Run in Colorado, 2014}

G. Clover, $\mathrm{MD}^{1}$; Laura Pyle, $\mathrm{PhD}^{2}$; Leo Lloyd, $\mathrm{RN}^{1}$

${ }^{1}$ Hardrock 100, ${ }^{2}$ School of Medicine, University of Colorado Denver

Objective.-To examine reported medical events, medical care provided, runner training, and racing characteristics at a high altitude ultramarathon run.

Methods.-Medical personnel reported all significant events to the Race Medical Director during and after the event. Significant medical events were followed up after the race by either the Medical Director or another race medical provider. Seventy-four runners completed an online postrace questionnaire regarding their experience, symptoms during and after the run, training characteristics, medication use, hydration and fueling strategy, and injuries both during the run and in prior ultramarathons. Associations between the medical problems experienced during the race and the runner's hydration, training, and experience were examined.

Results.-Fifty-four percent of runners reported fewer than ten $161-\mathrm{km}$ races, $51 \%$ had more than 20 years of running experience, and $>75 \%$ had more than 7 years of ultramarathon experience. Years of running and years of running ultras were not significantly associated with any of the medical outcomes. Peak training miles per week was significantly negatively correlated with shortness of breath $(P=.0316)$. Fifty percent of racers drank to thirst only, $39 \%$ on a time schedule and $16 \%$ by urine color or amount. The most frequent medical problems experienced during the race were nausea and vomiting (37\%), blisters $(34 \%)$, and shortness of breath $(24 \%)$. Twenty-three racers reported using pain medication during the race, mainly NSAIDs (nonsteroidal anti-inflammatory drugs). No definite serious altitude illness was reported despite the high altitude during much of the race. No runner received intravenous fluids during or after the race.

Conclusions. - This group of experienced runners appeared to tolerate high altitude within the race, by report. Medical issues were largely minor in nature. Running experience did not correlate strongly with reported symptoms. More study is needed, at this distance and altitude, regarding runner acclimatization practices, serum sodium, body weight, and ADH (antidiuretic hormone) levels as relates to hydration strategy and the possible effects of NSAIDs on the development of altitude illness.

\section{Utility of Urine Dipstick for Detecting Runners With} Acute Kidney Injury Following a 161-km Ultramarathon Morteza Khodaee, MD, MPH ${ }^{1}$; Bjørn Irion, $\mathrm{MD}^{2}$; Jack Spittler, MD, $\mathrm{MA}^{1}$; Martin D. Hoffman, $\mathrm{MD}^{3}$

${ }^{1}$ University of Colorado School of Medicine, ${ }^{2}$ Texas A\&M Health Science Center College of Medicine, ${ }^{3}$ University of California Davis Medical Center and Sacramento VA Medical Center

Objective.-To evaluate the incidence of acute kidney injury (AKI) during the Leadville 100 ultramarathon held at high altitude $(2800-3840 \mathrm{~m})$ and the utility of urine dipstick at detecting AKI.

Methods. - This was a prospective observational study. Blood and urine samples were collected from voluntary athletes before and immediately after the Leadville 100-mile race in August 2014.

Results.-Postrace dipstick urinalysis was done on 70 runners (51 finishers), and a postrace blood sample was obtained from 84 runners (61 finishers). Both prerace and postrace samples were collected from 37 participants, which were included in the analysis. AKI was defined as a serum creatinine increase ( $\geq 1.5$ times or $\geq 0.3 \mathrm{mg} / \mathrm{dL}$ from baseline) according to Kidney Disease: Improving Global Outcomes (KDIGO) Group. Eighteen runners $(48.6 \%)$ met the criteria for stage 1 AKI. Using a binary logistic regression, the only factors statistically correlated with likelihood of AKI were runners who lost more weight at the finish line and male gender $(P<.05$ and $P=.05$, respectively). Age, race time, race completion status, use of NSAIDs (nonsteroidal anti-inflammatory drugs) before or during the race, BMI (body mass index), and postrace creatine kinase and sodium levels were not correlated with presence of AKI. Using urine appearance (color $\geq 4$ out of 8 or not-clear) or specific gravity $(\geq 1.025)$ criteria predicted those meeting the stage 1 AKI criteria with sensitivity of $81.3 \%$ and specificity of $37.5 \%$. Using urine protein of at least $1+$ predicted those meeting the stage 1 AKI criteria with sensitivity of $43.8 \%$ and specificity of $87.5 \%$.

Conclusions.- Incidence of stage 1 AKI was relatively high in this ultramarathon. There was a higher rate of AKI among those who lost more weight at the finish line and male runners. Urine dipstick may be a useful screening tool for AKI.

We thank the Leadville Race Series. Funded in part by the ACSM Clinical Sports Medicine Endowment Grant. 